\title{
Lessons Learnt from a Decade of the Irish Hip Fracture Database
}

\author{
Helena Ferris ${ }^{\mathrm{a}}$ Louise Brent ${ }^{\mathrm{b}}$ Conor Hurson $^{\mathrm{c}}$ Emer Ahern $^{\mathrm{d}}$ \\ ${ }^{a}$ Department of Public Health, Health Service Executive-South, Cork, Ireland; b National Office of Clinical Audit, \\ Dublin, Ireland; 'Department of Trauma and Orthopaedics, St Vincent's University Hospital, Dublin, Ireland; \\ ${ }^{\mathrm{d}}$ Department of Geriatric Medicine, Cork University Hospital, Cork, Ireland
}

\section{Dear Editor,}

The Irish Hip Fracture Database (IHFD) is a clinical information system designed to acquire, store and evaluate clinical data. It was established in 2012 and over the last decade the IHFD has emerged as a central independent driving force for better patient outcomes in Ireland. It provides a reliable infrastructure for the collection of high-quality data, which are measured against explicit national standards of care. Since its inception, the IHFD has collected data on $>25,000$ hip fracture patients. These comprehensive data are available to those who use, manage, and deliver healthcare to refine clinical pathways and improve patient outcomes.

The IHFD is a meaningful collaboration between clinicians, health care professionals and policy makers. It is clinically endorsed and supported by the Irish Gerontological Society and the Irish Institute of Trauma and Orthopaedic Surgery, with operational management provided by the National Office for Clinical Audit (NOCA). All eligible 16 trauma units in Ireland submit data to the IHFD and there is regular 2-way feedback between participating hospitals, audit coordinators, clinical leads, governance committee and NOCA. Quarterly reports serve to benchmark the performance of participating hos- pitals in relation to others nationally and identify areas of excellence and opportunities for improvement. Annual reports are shared with senior decision makers in the Health Service Executive and Department of Health, with recommendations and actions agreed for implementation at a national and local level.

The maturity of the IHFD has led to several health system changes. Most notably, the IHFD has been instrumental in the development of orthogeriatric services in Ireland. This has consisted of several changes to the clinical and logistical elements of the hip fracture care pathway. During admission to an orthopaedic ward, patients with a hip fracture are now routinely assessed by a geriatrician. This is in comparison with the previous system where the Orthopaedic team would request a Geriatric Medicine consultation as required, which was a more reactive than proactive approach. Consultant or Registrar led ward rounds with support from Advance Nurse Practitioners in some sites, and multidisciplinary team meetings have greatly improved communication between teams and enhanced the service for the benefit of patients. Orthogeriatric models of care have been shown to improve functional outcomes for hip fracture patients, reduce length of stay and im- 
prove compliance with best practice guidelines [1]. Currently, a consensus model of orthogeriatric care for Ireland is being developed. However, more work is needed as these changes have not been uniformly implemented across all participating hospitals, primarily due to lack of funding for orthogeriatric posts. The IHFD will continue to advocate for the importance of an orthogeriatric model of care and provide the data to demonstrate its impact on patient care.

The IHFD was a key partner in the development of a "Trauma System for Ireland," the national report of the trauma steering group for the reconfiguration of trauma services in Ireland. This strategic report recognizes the importance of a multidisciplinary orthogeriatric service in each trauma unit, which is shown to significantly reduce mortality and improve patient outcomes in a costeffective manner [2]. Furthermore, the IHFD has been a key driver of the implementation of the Trauma care pathway and Hip Fracture Bypass protocol, which advocates for a 7 days a week service for trauma patients, with prompt access to theatre, medical support and physiotherapists.

Perhaps one of the most interesting lessons over the lifetime of the IHFD is although process measures are an important component of patient care, it is the simple interventions such as getting patients up and moving postoperatively that have the biggest impact on outcomes. A recent analysis of the IHFD by Ferris et al. [3] demonstrated that patients who were not mobilized on the day of or after surgery were $46 \%$ more likely to die in the hospital following hip fracture compared to those mobilized early (OR 1.46, 95\% CI: $1.25-1.70, p<0.001$ ). This finding led to the addition of one new formal standard of care in the Irish Hip Fracture Standards, in keeping with international best practice. This illustrates the value of having timely and accurate data to identify the parameters of care that have a real impact on patient outcomes and in turn inform the standards of care.

Tangible benefits have been seen since the establishment of the IHFD. More specifically, there has been:

- An increase in the proportion of patients seen by a geriatrician during admission from $11 \%$ to $82 \%$.

- An increase in specialist falls assessments from $47 \%$ to $85 \%$.

- More patients operated on within $48 \mathrm{~h}$ of admission, an increase from $69 \%$ to $75 \%$.

- A new standard of care for early mobilization, with $78 \%$ of patients now being stood out of bed at minimum on the day after surgery.

- Data completeness has increased to $99 \%$ [4].
In 2018, the IHFD introduced the Best Practice Tariff (BPT), a financial incentive for hospitals to provide highquality care to hip fracture patients. Payments of 1,000 EUR are issued to participating hospitals for each patient who receives care in keeping with all 9 of the Irish Hip Fracture Standards. This funding is ring fenced for the support of the trauma service in the receiving hospital. In the first year of the BPT, 278,000 EUR was paid out to participating hospitals, and this increased to 548,000 EUR in 2019 and to 710,000 EUR in 2020 [4]. The BPT has had a positive impact on the healthcare system as steady improvements in the proportion of patients receiving the best practice standards of care have been seen year on year. The substantial amount of money paid out has been used to purchase orthopaedic chairs for patients, electric beds, hoists and bladder scanners as well as funding staff training and quality improvement projects.

There are many exciting developments on the horizon for the IHFD. The scope of data collection is expanding to include longer term outcomes such as quality of life, mobility and survival up to 1 year after fracture, as part of the HipFORGE research project. Meaningful engagement with patients is a core value of the IHFD, and this qualitative approach will complement the extensive quantitative data already collected by the IHFD. The IHFD will continue to collaborate internationally with other Hip Fracture Audits and when necessary will produce rapid learning reports as seen during the COVID pandemic. A renewed focus will be placed on performing nutrition and delirium screens on all hip fracture patients, as well as avoiding repeated fasting of patients. Care bundles will be developed to streamline the patient journey from pre-hospital to rehabilitation with more community integration.

It is clear that the IHFD is a vehicle for change and a catalyst for improvement by facilitating evidence-based decision-making. As Ireland's trauma system continues to evolve, the IHFD is in prime position to monitor the impact of the reconfiguration of services. It is imperative that this rich data source is utilized to optimize clinical care, governance and outcomes for hip fracture patients.

\section{Acknowledgments}

The authors would like to acknowledge the continuing support and dedication of all the IHFD audit coordinators and clinical leads, Healthcare Pricing Office, Irish Hip Fracture Database Governance Committee, Irish Institute of Trauma and Orthopaedic Surgery, Irish Gerontological Society, Health Service Executive - Quality Improvement Team and the National Office of Clinical Audit. 


\section{Conflict of Interest Statement}

The authors have no conflicts of interest to declare.

\section{Funding Sources}

No funding, grants, or financial support was received for the preparation of this manuscript.

\section{Author Contributions}

H.F. conceptualized and designed the manuscript. H.F., L.B., C.H., and E.M. drafted the manuscript, provided feedback, and approved the final draft.

\section{References}

1 Murphy RP, Reddin C, Murphy EP, Waters R, Murphy CG, Canavan M. Key service improvements after the introduction of an integrated Orthogeriatric service. Geriatr Orthop Surg Rehabil. 2019;10:2151459319893898.

2 A Trauma System for Ireland; Report of the Trauma Steering Group. Department of Health; 2018. Available from: www.gov.ie. Accessed 2021 Oct 22.
3 Ferris H, Brent L, Coughlan T. Early mobilisation reduces the risk of in-hospital mortality following hip fracture. Eur Geriatr Med. 2020 Aug;11(4):527-33.

4 National Office of Clinical Audit; Irish hip fracture database national report. Dublin: National Office of Clinical Audit; 2020. ISSN 2565-5388. Available from: https://www. noca.ie/audits/irish-hip-fracture-database. Accessed 2021 Oct 21 